\title{
REGULARIZED LINEAR PROGRAMS WITH EQUILIBRIUM CONSTRAINTS *
}

\author{
O. L. MANGASARIAN ${ }^{\dagger}$
}

\begin{abstract}
A bstract. We consider an arbitrary linear program with equilibrium constraints (LPEC) that may possibly be infeasible or have an unbounded objective function. We regularize the LPEC by perturbing it in a minimal way so that the regularized problem is solvable. We show that such regularization leads to a problem that is guaranteed to have a solution which is an exact solution to the original LPEC if that problem is solvable, otherwise it is a residual-minimizing approximate solution to the original LPEC. We propose a finite successive linearization algorithm for the regularized problem that terminates at point satisfying the minimum principle necessary optimality condition for the problem.
\end{abstract}

Key words. Linear programs with equilibrium constraints, regularization, exact penalty, concave minimization.

AMS subject classifications. 90C05, 90C30

1. Introduction. We consider the following linear program with equilibrium constraints:

$$
\begin{aligned}
& \min _{x, y} c^{T} x+d^{T} y \\
& \text { s.t. } A x+B y-b \geq 0 \\
& \begin{array}{l}
0 \leq y \perp \quad N x+M y-q \geq 0 \\
x \quad y \quad \geq 0
\end{array}
\end{aligned}
$$

where $A \in R^{p \times n}, B \in R^{p \times m}, N \in R^{m \times n}$, and $M \in R^{m \times m}$, are given matrices, $c \in R^{n}, d \in R^{m}, b \in R^{p}$, and $q \in R^{m}$, are given vectors, and $\perp$ denotes orthogonality, that is the scalar product of the two vectors appearing on either side of $\perp$ is zero. This is a special case of a mathematical program with equilibrium constraints [7] that has important applications in machine learning [9, 11,3]. Fukushima and Pang [6] were the first to address the feasibility issue for a mathematical program with equilibrium constraints (MPEC) similar to those of our LPEC (1.1) and point out that it is a difficult problem in general. They gave sufficient conditions to ensure feasibility, which are not trivial to check, but did not address the question of handling possibly infeasible constraints. In the present work we wish to address this case as well as the more general problem of an unsolvable LPEC via an exact penalty regularization approach. We note that exact penalty approaches have been proposed for solvable LPECs in [9] and for solvable MPECs in [15].

We briefly outline the contents of the paper now. In Section 2 we give a general exact penalty result which shows that a fixed solution of a penalty problem, for an increasing sequence of penalty parameters tending to infinity, minimizes the penalty term. Such a solution also minimizes the objective function of the original unpenalized, possibly infeasible, problem over the set of minimizers of the penalty term. If the penalty term minimum is zero then the original unpenalized problem is feasible and solvable and a solution to it is obtained by the penalty function minimization.

*This work was supported by National Science Foundation Grant CCR-9322479 and Air Force Office of Scientific Research Grant F49620-97-1-0326 as Mathematical Programming Technical Report 97-13, November 1997. Revised January 1998.

${ }^{\dagger}$ Computer Sciences Department, University of Wisconsin, 1210 West Dayton Street, Madison, WI 53706,olvi@cs.wisc.edu. 
In Section 3 we apply the penalty result to regularize the LPEC (1.1) and show that solving the regularized penalty problem, which consists of minimizing a piecewiselinear concave function on a polyhedral set, for any value of the penalty parameter exceeding some threshold, leads to a residual-minimizing solution to the LPEC which is an exact solution if the LPEC is solvable. In Section 4 we propose a supergradientbased successive linearization algorithm, that terminates in a finite number of steps at a stationary point, for solving the regularized penalty problem for the LPEC. A similar supergradient-based algorithm has been proposed for a general linear complementarity problem [14], and successfully used on the NP-complete knapsack feasibility problem [10].

1.1. Notation and Background. A word about our notation and background material. All vectors will be column vectors unless transposed to a row vector by a prime superscript ${ }^{T}$. The scalar product of two vectors $x$ and $y$ in the $n$-dimensional real space $R^{n}$ will be denoted by $x^{T} y$. For a mathematical program $\min _{x \in X} f(x)$, where $f: R^{n} \longrightarrow R$, the notation arg $\min _{x \in X} f(x)$, will denote the set of solutions of the mathematical program $\min _{x \in X} f(x)$, while arg vertex $\min _{x \in X} f(x)$ will denote the set of vertex solutions of the same problem when $X$ is polyhedral. For $x \in R^{n}$ and $p \in$ $[1, \infty)$, the norm $\|x\|_{p}$ will denote the $p$-norm: $\left(\sum_{i=1}^{n}\left|x_{i}\right|^{p}\right)^{\frac{1}{p}}$ and $\|x\|_{\infty}$ will denote $\max _{1 \leq i \leq n}\left|x_{i}\right|$. For $x \in R^{n},\left(x_{+}\right)_{i}=\max \left\{0, x_{i}\right\}, i=1, \ldots, n$. For an $m \times n$ matrix $A, A_{i}$ will denote row $i$ of $A$ and $A_{j}$ will denote column $j$ of $A$. The identity matrix in a real space of arbitrary dimension will be denoted by $I$, while a column vector of ones of arbitrary dimension will be denoted by $e$ and a column vector of zeros of arbitrary dimension will be denoted by 0 . The symbol $:=$ will denote a definition of the term appearing to the left of the symbol by the term appearing to the right of the symbol. For a set $X \in R^{n} \tilde{X}$ will denote its complement in $R^{n}$.

2. Exact Penalty for Possibly Infeasible Problems. We derive in this section an exact penalty formulation for a possibly infeasible mathematical program and show that such an exact penalty approach can yield an infeasibility-minimizing solution to the problem for the infeasible case and an exact solution for the solvable case. We state this result in the following theorem which may be useful for handling general infeasible linear and nonlinear programs other than infeasible LPECs.

Theorem 2.1. Exact Penalty for Inconsistent Problems. Consider the possibly infeasible mathematical program $\min _{x \in S \cap T} f(x)$ where $f: \emptyset \neq S \subset R^{n} \rightarrow R$ and $T \subset R^{n}$. Let the penalty $Q: S \rightarrow R$ be defined such that:

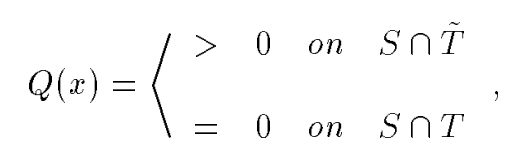

and let

$$
P(x, \alpha):=f(x)+\alpha Q(x), \quad \alpha \geq 0 .
$$

If for a sequence of positive numbers $\left\{\alpha^{i}\right\}_{i=0}^{\infty} \uparrow \infty$ :

$$
\bar{x} \in \arg \min _{x \in S} P\left(x, \alpha^{i}\right), \quad i=0,1, \ldots
$$

then: 
(i) $Q(\bar{x})=\inf _{x \in S} Q(x) \geq 0$.

(ii) $\bar{x} \in \arg \min _{x \in S} P(x, \alpha), \forall \alpha \geq \alpha^{0}$.

(iii) $\bar{x} \in \arg \min _{x \in S}\left\{f(x) \mid Q(x)=\inf _{x \in S} Q(x)\right\}$.

(iv) If $\inf _{x \in S} Q(x)=0$, then $\bar{x} \in \arg \min _{S \cap T} f(x)$.

Proof

(i) Let $\bar{Q}=\inf _{x \in S} Q(x) \geq 0$. Suppose $Q(\bar{x})>\bar{Q}$ and we will exhibit a contradiction and hence $Q(\bar{x})=\bar{Q}$. To show a contradiction let:

$$
\epsilon=\frac{Q(\bar{x})-\bar{Q}}{4}>0, x \in S, \text { such that } Q(x)<\bar{Q}+\epsilon,
$$

and choose $\alpha^{\bar{i}}$ such that:

$$
\alpha^{\bar{i}}>\max \left\{\frac{f(x)-f(\bar{x})}{Q(\bar{x})-\bar{Q}-2 \epsilon}, \alpha^{0}\right\} .
$$

We then have the following contradiction:

$$
\begin{aligned}
& f(x)+\alpha^{\bar{i}} \bar{Q}+\alpha^{\bar{i}} \epsilon>f(x)+\alpha^{\bar{i}} Q(x)=P\left(x, \alpha^{\bar{i}}\right) \geq \\
& P\left(\bar{x}, \alpha^{\bar{i}}\right)=f(\bar{x})+\alpha^{\bar{i}} Q(\bar{x})>f(x)+\alpha^{\bar{i}} \bar{Q}+2 \alpha^{\bar{i}} \epsilon,
\end{aligned}
$$

where the last inequality follows from the choice of $\alpha^{\bar{i}}$.

(ii) Since

$$
f(x)-f(\bar{x})+\alpha^{0}(Q(x)-Q(\bar{x})) \geq 0, \quad \forall x \in S,
$$

it follows, since $Q(x)-Q(\bar{x}) \geq 0, \forall x \in S$, that:

$$
f(x)-f(\bar{x})+\alpha(Q(x)-Q(\bar{x})) \geq 0, \forall x \in S, \forall \alpha \geq \alpha^{0} .
$$

Hence

$$
P(x, \alpha) \geq P(\bar{x}, \alpha), \forall x \in S, \forall \alpha \geq \alpha^{0} .
$$

(iii) Suppose that $x \in S$ and $Q(x)=Q(\bar{x})$. Then

$$
\begin{aligned}
& f(x)=f(x)+\alpha^{0}(Q(x)-Q(x))=P\left(x, \alpha^{0}\right)-\alpha^{0} Q(x) \geq \\
& P\left(x, \alpha^{0}\right)-\alpha^{0} Q(\bar{x}) \geq P\left(\bar{x}, \alpha^{0}\right)-\alpha^{0} Q(\bar{x})=f(\bar{x}) .
\end{aligned}
$$

Hence,

$$
\bar{x} \in \arg \min _{x \in S}\left\{f(x) \mid Q(x) \leq Q(\bar{x})=\inf _{x \in S} Q(x)\right\} .
$$

(iv) This follows directly from (iii) and the definition (2.1) of $Q(x)$ which implies that $x \in T$ if $\inf _{x \in S} Q(x)=0 . \square$

REMARK 2.2. For special classes of important problems that arise in machine learning and data mining [11, 2, 13] where $P(x, \alpha)$ is concave in $x$ and $S$ is a polyhedral set without straight lines going to infinity in both directions, such as problem (3.8) below, the point $\bar{x}$ of Theorem 2.1 is any repeated vertex solution of $\min _{x \in S} P\left(x, \alpha^{i}\right),\left\{\alpha^{i}\right\}_{i=0}^{\infty} \uparrow \infty$. Although it is not easy to get such a globally optimal 
repeated vertex, a stationary repeated vertex can be easily obtained by successive linearization algorithms such as Algorithm 4.1 below. Such stationary vertices turn out to be very useful in machine learning and data mining applications [11, 2, 13].

In a related weaker result, Tikhonov and Arsenin [19, Theorem 1, p 101] establish asymptotic convergence of an inexact quadratic penalty function solution for the very special case of projecting a point on a possibly empty solution set of a system of linear equations. They show convergence to a projection of the point onto the set of solutions of the normal equations of the linear system when the linear system is infeasible. In contrast for example, Part (iii) of the above theorem shows how the Big M Method of linear programming [17, pp 81-82] can terminate at an optimal solution over the set of infeasibility minimizers when a linear program is infeasible.

We turn our attention now to LPECs that may possibly be infeasible or whose objective may be unbounded.

3. LPEC Regularization. We consider now the LPEC (1.1) with no assumptions on feasibility or boundedness of the objective function to start with. We first regularize the underlying linear program to the LPEC (1.1), that is problem (1.1) with the complementarity condition removed, and make it solvable in case it is not. Hence if the underlying linear program to the LPEC (1.1) is solvable or regularized to be so, the LPEC (1.1) will have a bounded objective function if it is feasible. This is is so because the feasible region of the LPEC (1.1) is a subset of the feasible region of the underlying linear program, and both problems have the same objective function. This preliminary regularization can be achieved [12, Theorem 2.2] by appropriately modifying the underlying linear program by solving:

$$
\min _{z}\left\|z-(z-H z-h)_{+}\right\|_{1}
$$

which is a regularization of the linear complementarity problem associated with the linear program underlying LPEC (1.1):

$$
0 \leq z \perp H z+h \geq 0
$$

Here

$$
H=\left[\begin{array}{cccc}
0 & 0 & -A^{T} & -N^{T} \\
0 & 0 & -B^{T} & -M^{T} \\
A & B & 0 & 0 \\
N & M & 0 & 0
\end{array}\right], h=\left[\begin{array}{c}
c \\
d \\
-b \\
-q
\end{array}\right], \quad z=\left[\begin{array}{l}
x \\
y \\
u \\
v
\end{array}\right]
$$

We note that because of the skew symmetry of $H$, the regularization problem (3.1) can be rewritten as a linear program and its solution can be used to generate a solvable underlying linear program for LPEC (1.1) [12, Equation (9)]. For further details about this regularization we refer the interested reader to [12, Theorem 2.2]. Another and simpler regularization of the linear program underlying LPEC (1.1) is one where the coefficients $c, d, b, q$ of LPEC (1.1) are replaced by $\bar{c}, \bar{d}, \bar{b}, \bar{q}$ as follows:

$$
\bar{c}=c+\bar{r}_{1}, \bar{d}=d+\bar{r}_{2}, \bar{b}=b-\bar{s}_{1}, \bar{q}=q-\bar{s}_{2},
$$


where $\left(\bar{s}_{1}, \bar{s}_{2}\right)$ and $\left(\bar{r}_{1}, \bar{r}_{2}\right)$ are solutions of the following "Phase I" primal and dual linear programs:

$$
\left(\bar{x}, \bar{y}, \bar{s}_{1}, \bar{s}_{2}\right) \in \arg \min _{x, y, s_{1}, s_{2}}\left\{\begin{array}{l|l}
e^{T} s_{1}+e^{T} s_{2} & \begin{array}{l}
A x+B y+s_{1} \geq b \\
N x+M y+s_{2} \geq q \\
\left(x, y, s_{1}, s_{2}\right) \geq 0
\end{array}
\end{array}\right\}
$$

$$
\left(\bar{u}, \bar{v}, \bar{r}_{1}, \bar{r}_{2}\right) \in \arg \min _{u, v, r_{1}, r_{2}}\left\{\begin{array}{l|l}
e^{T} r_{1}+e^{T} r_{2} & \begin{array}{l}
A^{T} u+N^{T} v-r_{1} \leq c, \\
B^{T} u+M^{T} v-r_{2} \leq d \\
\left(u, v, r_{1}, r_{2}\right) \geq 0
\end{array}
\end{array}\right\}
$$

We note that for LPECs arising in machine learning [9, 11,3] there is no need for this preliminary regularization in as much as the underlying linear programs there are feasible and solvable. For the rest of the paper we shall assume that such a regularization as given by replacing $c, d, b, q$ by $\bar{c}, \bar{d}, \bar{b}, \bar{q}$ of (3.4) has been either carried out or is un-necessary and for simplicity of notation we shall not replace $c, d, b, q$ by $\bar{c}, \bar{d}, \bar{b}, \bar{q}$ as would be required if such a regularization takes place. Note that this preliminary regularization, which renders the underlying linear program solvable, does in no way ensure that the LPEC is solvable. It was pointed out in [6] that such feasibility of the underlying linear program does not ensure feasibility of the LPEC constraints. In fact one of the main objectives of this work is the ability to handle LPECs that may not have a solution. Hence starting with an LPEC (1.1) for which the underlying linear program is solvable, either through a preliminary regularization as described above or because it is naturally so, we define a regularized problem which is guaranteed to be solvable as follows. (We note in passing that we can forgo this preliminary regularization and pass on the regularization process in its entirety to one LPEC regularization process which would be considerably more complicated than the proposed one. For the sake of simplicity and because machine learning LPECs need no such preliminary regularization, we opt for the simpler regularization presented below.)

We define now two sets associated with the LPEC (1.1) which will be used in applying Theorem 2.1 to obtain our principal result.

$$
\begin{aligned}
& S:=\{(x, y) \mid A x+B y \geq b, N x+M y \geq q,(x, y) \geq 0\} \\
& T:=\{(x, y) \mid y \perp N x+M y-q\} .
\end{aligned}
$$

We state our principal regularization result now.

Theorem 3.1. LPEC Regularization Let $S$ be nonempty, let $c^{T} x+d^{T} y$ be bounded below on $S$ and consider the following penalty problem:

$$
\min _{(x, y) \in S} c^{T} x+d^{T} y+\alpha e^{T} \min \{y, N x+M y-q\}, \alpha \geq 0
$$

Then the following hold:

(i) The penalty problem (3.7) has a vertex solution for each $\alpha \geq 0$.

(ii) There exist $\bar{x}$ and $\alpha^{0} \geq 0$ such that for all $\alpha \geq \alpha^{0}$

$$
\bar{x} \in \arg \text { vertex } \min _{(x, y) \in S} c^{T} x+d^{T} y+\alpha e^{T} \min \{y, N x+M y-q\}
$$

(iii) Furthermore, each $\bar{x}$ that satisfies (ii) solves the following residual-minimizing problem:

$$
\min _{(x, y)}\left\{c^{T} x+d^{T} y \mid(x, y) \in \arg \min _{(x, y) \in S} e^{T} \min \{y, N x+M y-q\}\right\}
$$


(iv) If $\min _{(x, y) \in S} e^{T} \min \{y, N x+M y-q\}=0$ then each $\bar{x}$ that satisfies (ii) is an exact solution of the LPEC (1.1).

\section{Proof}

(i) Since the concave objective function of the penalty problem (3.7) is bounded below on the nonempty polyhedral region that contains no lines that go to infinity in both directions, it must by [18, Corollary 32.3.4] have a vertex solution.

(ii) Since $S$ has a finite number of vertices, then there exists a sequence of positive numbers $\left\{\alpha^{i}\right\}_{i=0}^{\infty} \uparrow \infty$ such that:

$\bar{x} \in \arg$ vertex $\min _{x \in S} c^{T} x+d^{T} y+\alpha e^{T} \min \{y, N x+M y-q\}, \forall \alpha \in\left\{\alpha^{i}\right\}_{i=0}^{\infty} \uparrow \infty$.

The result now follows from Theorem 2.1(i)-(ii).

(iii) Follows from Theorem 2.1(iii).

(iv) Follows from Theorem 2.1(iv).

It is interesting to note that the penalty term that multiplies the parameter $\alpha$ in the penalty problem (3.7) is the natural error residual for the complementarity problem $0 \leq y \perp N x+M y-q \geq 0$ that appears in the constraints of LPEC (1.1) and constitutes a local error bound for the general linear complementarity problem, and a global error bound for $M \in R_{0}$, the class of matrices $M$ for which 0 is the unique solution to the homogeneous linear complementarity problem: $0 \leq y \perp M y \geq 0$ $[8,16]$.

We turn our attention now to solution methods for the regularized problem.

4. Successive Linearization Algorithm. We propose here a finite successive linearization algorithm introduced in [14] that utilizes a supergradient of the piecewiselinear concave objective function of the penalty problem (3.7) as follows.

Algorithm 4.1. Successive Linearization Algorithm Choose $\alpha>0$. Start with an arbitrary $\left(x^{0}, y^{0}\right) \in R^{n+m}$. Having $\left(x^{i}, y^{i}\right)$ determine $\left(x^{i+1}, y^{i+1}\right)$ as follows:

$\left(x^{i+1}, y^{i+1}\right) \in \arg$ vertex $\min _{(x, y) \in S}\left\{\begin{array}{l}c^{T}\left(x-x^{i}\right)+d^{T}\left(y-y^{i}\right) \\ +\alpha\left(\partial_{x} \phi\left(x^{i}, y^{i}\right)^{T}\left(x-x^{i}\right)+\partial_{y} \phi\left(x^{i}, y^{i}\right)^{T}\left(y-y^{i}\right)\right)\end{array}\right\}$,

where $\phi(x, y)$ is the penalty term of (3.7), that is:

$$
\phi(x, y):=e^{T} \min \{y, N x+M y-q\},
$$

and $\partial_{x} \phi(x, y), \partial_{y} \phi(x, y)$ are supergradients of $\phi(x, y)$ with respect to $x$ and $y$ respectively, that is:

$$
\begin{aligned}
& \partial_{x} \phi(x, y)=\sum_{j=1}^{m}\left\langle\begin{array}{l}
0 \text { if } y_{j}<N_{j} x+M_{j} y-q_{j} \\
\left(1-\lambda_{j}\right) 0+\lambda_{j} N_{j} \text { if } y_{j}=N_{j} x+M_{j} y-q_{j}, 0 \leq \lambda_{j} \leq 1 \\
N_{j} \text { if } y_{j}>N_{j} x+M_{j} y-q_{j}
\end{array}\right\rangle \\
& \partial_{y} \phi(x, y)=\sum_{j=1}^{m}\left\langle\begin{array}{l}
I_{j} \text { if } y_{j}<N_{j} x+M_{j} y-q_{j} \\
\left(1-\lambda_{j}\right) I_{j}+\lambda_{j} M_{j} \text { if } y_{j}=N_{j} x+M_{j} y-q_{j}, 0 \leq \lambda_{j} \leq 1 \\
M_{j} \text { if } y_{j}>N_{j} x+M_{j} y-q_{j}
\end{array}\right\rangle
\end{aligned}
$$

Stop if $\left(x^{i}, y^{i}\right)$ is a solution of (4.1).

By using [14, Theorem 3] finite termination of the above algorithm can be established as follows. 
Theorem 4.2. Finite Termination The Successive Linearization Algorithm 4.1 generates a finite sequence of points with strictly decreasing objective function values for the penalty problem (3.7). The sequence terminates at a point $\left(x^{\bar{i}}, y^{\bar{i}}\right) \in S$ that satisfies the minimum principle necessary optimality condition for (3.7):

$c^{T}\left(x-x^{\bar{i}}\right)+d^{T}\left(y-y^{\bar{i}}\right)+\alpha\left(\partial_{x} \phi\left(x^{\bar{i}}, y^{\bar{i}}\right)^{T}\left(x-x^{\bar{i}}\right)+\partial_{y} \phi\left(x^{\bar{i}}, y^{\bar{i}}\right)^{T}\left(y-y^{\bar{i}}\right)\right) \geq 0, \quad \forall(x, y) \in S$. (4.4)

We note that the bilinear algorithm of [10] for solving the knapsack feasibility problem as a linear complementarity problem can be interpreted as a special case of Algorithm 4.1 with a fixed $\lambda_{j}=0$. That bilinear algorithm solved 80 consecutive instances of the knapsack LCP ranging in size between 10 and 3000 without failure. This is an indication that the proposed Algorithm 4.1 may be effective for classes of LPECs.

5. Summary and Future Work. We have proposed an always-solvable regularization of a completely general LPEC. The regularized problem is a piecewise linear concave minimization problem on a polyhedral set that generates a residualminimizing solution to the original LPEC which is an exact solution if the LPEC is solvable. A finite successive linearization algorithm is proposed for solving the regularized problem that terminates at a stationary point. A smoothing of the penalty problem (3.7) objective function using a smoothing of the plus-function proposed in $[4,5]$ can also be used and can be shown to lead to an exact solution the penalty problem (3.7) for a finite value of the penalty parameter as was done in [1]. The encouraging computational results of [10] on a special case of the proposed algorithm on an NP-hard problem is a possible indicator of the possible effectiveness of the proposed algorithm for solving the regularized LPEC. 


\section{REFERENCES}

[1] P. S. Bradley, O. L. Mangasarian, and J. B. Rosen. Parsimonious least norm approximation. Technical Report 97-03, Computer Sciences Department, University of Wisconsin, Madison, Wisconsin, March 1997. Computational Optimization and Applications, to appear. ftp://ftp.cs.wisc.edu/math-prog/tech-reports/97-03.ps.Z.

[2] P. S. Bradley, O. L. Mangasarian, and W. N. Street. Feature selection via mathematical programming. INFORMS Journal on Computing, 1998. To appear. Available at ftp://ftp.cs.wisc.edu/math-prog/tech-reports/95-21.ps.Z.

[3] E. J. Bredensteiner and K. P. Bennett. Feature minimization within decision trees. Department of Mathematical Sciences Math Report No. 218, Rensselaer Polytechnic Institute, Troy, NY 12180, 1995. Computational Optimizations and Applications. to appear.

[4] Chunhui Chen and O. L. Mangasarian. Smoothing methods for convex inequalities and linear complementarity problems. Mathematical Programming, 71(1):51-69, 1995.

[5] Chunhui Chen and O. L. Mangasarian. A class of smoothing functions for nonlinear and mixed complementarity problems. Computational Optimization and Applications, 5(2):97-138, 1996.

[6] M. Fukushima and J.-S. Pang. Some feasibility issues in mathematical programs with equilibrium constraints. Technical report, Department of Mathematics \& Physics, Johns Hopkins University, January 1997. SIAM Journal on Optimization, to appear.

[7] Z.-Q. Luo, J.-S. Pang, and D. Ralph. Mathematical Programs with Equilibrium Constraints. Cambridge University Press, Cambridge, England, 1996.

[8] Z.-Q. Luo and P. Tseng. Error bound and convergence analysis of matrix splitting algorithms for the affine variational inequality problem. SIAM Journal on Optimization, 2:43-54, 1992.

[9] O. L. Mangasarian. Misclassification minimization. Journal of Global Optimization, 5:309-323, 1994.

[10] O. L. Mangasarian. The linear complementarity problem as a separable bilinear program. Journal of Global Optimization, 6:153-161, 1995.

[11] O. L. Mangasarian. Machine learning via polyhedral concave minimization. In H. Fischer, B. Riedmueller, and S. Schaeffler, editors, Applied Mathematics and Parallel ComputingFestschrift for Klaus Ritter, pages 175-188. Physica-Verlag A Springer-Verlag Company, Heidelberg, 1996. Available at ftp://ftp.cs.wisc.edu/math-prog/tech-reports/95-20.ps.Z.

[12] O. L. Mangasarian. The ill-posed linear complementarity problem. In Michael Ferris and JongShi Pang, editors, Complementarity and Variational Problems, pages 226-233. SIAM, Philadelphia, PA, 1997. Available at ftp://ftp.cs.wisc.edu/math-prog/tech-reports/9515.ps.Z.

[13] O. L. Mangasarian. Mathematical programming in data mining. Data Mining and Knowledge Discovery, 1(2):183-201, 1997. Available at ftp://ftp.cs.wisc.edu/math-prog/techreports/96-05.ps.Z.

[14] O. L. Mangasarian. Solution of general linear complementarity problems via nondifferentiable concave minimization. Acta Mathematica Vietnamica, 22(1):199-205, 1997. Available at ftp://ftp.cs.wisc.edu/math-prog/tech-reports/96-10.ps.Z.

[15] O. L. Mangasarian and J.-S. Pang. Exact penalty functions for mathematical programs with linear complementarity constraints. Optimization, 42:1-8, 1997. Available from: ftp://ftp.cs.wisc.edu/math-prog/tech-reports/96-06.ps.Z.

[16] O. L. Mangasarian and J. Ren. New improved error bounds for the linear complementarity problem. Mathematical Programming, 66:241-255, 1994.

[17] K. G. Murty. Linear Programming. John Wiley \& Sons, New York, 1983.

[18] R. T. Rockafellar. Convex Analysis. Princeton University Press, Princeton, New Jersey, 1970.

[19] A. N. Tikhonov and V. Y. Arsenin. Solutions of Ill-Posed Problems. John Wiley \& Sons, New York, 1977 . 